Dennis Gratzfeld, Juliane Heitkämper, Julien Debailleul and Matthias Olzmann*

\title{
On the influence of water on urea condensation reactions: a theoretical study
}

https://doi.org/10.1515/zpch-2020-1658

Received March 26, 2020; accepted April 30, 2020

Abstract: The influence of water molecules on the kinetics of urea condensation reactions was studied with high-level quantum chemical methods and statistical rate theory. The study focuses on the production of biuret, triuret, and cyanuric acid from urea because of their relevance as unwanted byproducts in the ureabased selective catalytic reduction (urea-SCR) exhaust after treatment of Diesel engines. In order to characterize the potential energy surfaces and molecular reaction pathways, calculations with explicitly-correlated coupled-cluster methods were performed. It turned out that the reactions proceed via pre-reactive complexes and the inclusion of one or two water molecules into the condensation mechanisms leads to a decrease of the energy barriers. This effect is particularly pronounced in the production of biuret. Due to the pre-reactive equilibria, the rates of the overall reactions can increase or decrease by incorporating water into the mechanism, depending on the temperature and water concentration. Under the conditions of urea-SCR, the studied reactions are too slow to contribute to the observed byproduct formation.

Keywords: biuret; cyanuric acid; triuret; urea; urea-SCR; water catalysis.

\section{Introduction}

Urea and its condensation products occur in many biological and technical processes. These range from mammalian metabolism via use of nitrogen fertilizers in agriculture to exhaust gas after treatment in automotive applications.

Dedicated to Prof. Dr. Friedrich Temps on the occasion of his 65th birthday.

*Corresponding author: Matthias Olzmann, Institut für Physikalische Chemie, Karlsruher Institut für Technologie (KIT), Kaiserstr. 12, 76131, Karlsruhe, Germany,

E-mail: matthias.olzmann@kit.edu

Dennis Gratzfeld, Juliane Heitkämper and Julien Debailleul: Institut für Physikalische Chemie, Karlsruher Institut für Technologie (KIT), Kaiserstr. 12, 76131 Karlsruhe, Germany 
Condensation reactions of urea play a particular role in the urea-based selective catalytic reduction (urea-SCR), which is used to reduce $\mathrm{NO}_{x}$ emissions from diesel cars. In the urea-SCR process (see e. g. [1, 2]), a urea-water solution is injected into the exhaust tract of a diesel engine to in situ produce ammonia that reduces $\mathrm{NO}_{x}$ to harmless $\mathrm{N}_{2}$ and $\mathrm{H}_{2} \mathrm{O}$ in a heterogeneously catalyzed reaction. Though the desired production of ammonia (and carbon dioxide) from the urea-water solution is the major reaction route, byproducts may be generated due to the condensation reactions. Under practical conditions, these byproducts can form solid deposits that may block the exhaust line or coat the catalyst, compromising the efficiency of the exhaust gas after treatment system.

In view of this serious impairment of a widely used technical process, byproduct formation in the thermolysis and hydrolysis of urea has been extensively discussed in the literature. Most of the works, however, were applicationoriented, investigating the effect on the exhaust gas catalyst system as a whole (see e. g. [3-5]). The modeling aspects in these studies were largely devoted to physical transport processes, and chemistry was included only in a few cases in the form of simple empirical chemical mechanisms [6-8]. In deposits obtained under practical operation conditions, biuret, triuret, cyanuric acid, and ammelide could be identified as major constituents (for structures see Figure 1) [9-11]. This finding is in line with results from a number of thermogravimetric/thermoanalytic studies on decomposing solid urea (see e. g. [7-14]).

Besides these recent, more application-oriented works, there are some early, more fundamental studies on the decomposition of urea in aqueous solution at temperatures between 273 and $373 \mathrm{~K}$. These investigations indicate that an ionic decomposition channel to give ammonium and isocyanate dominates the overall reaction [1519]. The characteristic reaction times in these studies, however, were on the order of hours that are several orders of magnitude larger than under exhaust tract conditions.

In contrast to the large number of experimental studies, and besides two recent theoretical works on thermodynamic quantities [20, 21], there appear to be only two theoretical works investigating elementary reaction steps in the formation of biuret, triuret, and cyanuric acid. Jeilani et al. [22] applied density functional<smiles>NC(=O)NC(N)=O</smiles>

biuret<smiles>NC(=O)NC(=O)NC(N)=O</smiles>

triuret

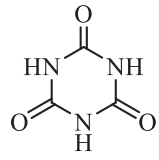

cyanuric acid<smiles>N=c1[nH]c(=O)[nH]c(=O)[nH]1</smiles>

ammelide

Figure 1: Products of urea condensation reactions. Note that in the present work, the keto forms of these compounds, which are generally the more stable tautomers in the gas phase, are considered. 
theory (DFT) to study the formation of $s$-triazines through low-temperature radical mechanisms in prebiotic systems, where radical production was assumed to be triggered by photolysis or discharge processes. Sebelius et al. [23] focused on SCR conditions and complemented their thermogravimetric experiments on the formation of biuret, triuret, and cyanuric acid by quantum chemical calculations. The authors considered reactions of closed-shell species in the gas phase in the absence of catalysts and calculated structures and energies of stationary points, using DFT at the B3PW91/6-31++ $\mathrm{G}^{\star \star}$ level. Qualitative conclusions regarding possible reaction pathways were drawn, but the obtained energy barriers appear too high to make these mechanisms competitive under SCR conditions. Kinetic data were not derived.

It is well known (see e. g. [2, 24]) that a critical intermediate in the formation of urea condensation products is isocyanic acid, which is produced together with ammonia in the primary decomposition step of urea, $\mathrm{H}_{2} \mathrm{NC}(\mathrm{O}) \mathrm{NH}_{2} \rightarrow \mathrm{HNCO}+\mathrm{NH}_{3}$. The addition of isocyanic acid to urea yields biuret, reaction 1, and the subsequent addition of isocyanic acid to biuret yields triuret, reaction 2 . The latter species can react to give cyanuric acid by cyclization and elimination of ammonia, reaction 3 [23]. Note that reactions 1-3 include an intramolecular hydrogen atom transfer.

$$
\begin{gathered}
\mathrm{HNCO}+\text { urea } \rightarrow \text { biuret } \\
\mathrm{HNCO}+\text { biuret } \rightarrow \text { triuret } \\
\text { triuret } \rightarrow \mathrm{NH}_{3}+\text { cyanuric acid }
\end{gathered}
$$

Because in the urea-SCR process a urea-water solution is used (see above), the question for the role of water in this reaction mechanism arises. From atmospheric chemistry (see e. g. [25]), it is well known that water can act as a catalyst in $\mathrm{H}$-atom transfer reactions also of closed-shell species such as the formation of sulfuric acid from $\mathrm{SO}_{3}$ and water [26, 27]. A quantum chemical investigation based on DFT by Kallies and Mitzner [28] was the first study to apply this concept to the urea reaction system, specifically to the thermal decomposition of urea according to $\mathrm{H}_{2} \mathrm{NC}(\mathrm{O})$ $\mathrm{NH}_{2} \rightarrow \mathrm{HNCO}+\mathrm{NH}_{3}$ in aqueous solution. These workers found a significant decrease of the energy barrier when one or several water molecules were involved in the $\mathrm{H}$-atom transfer. Several other quantum chemical studies on urea decomposition arrive at similar conclusions [29-34].

In the present work, this approach is applied to urea condensation reactions. The topic is restricted, however, to gas-phase mechanisms of closed-shell neutral species with water acting as a reaction partner. Reactions of ionic or radical species are not considered because these would form extensive topics of their own. For a recent study on HNCO hydrolysis, the reader is referred to [35]. 
We performed quantum chemical calculations, starting from the results of [23], but went beyond the DFT level used there. Though we used DFT to explore the potential energy surfaces, we included dispersion interactions and calculated the energies of the stationary points with coupled-cluster methods. Furthermore, the effect of including one or two water molecules in the elementary reaction mechanisms was studied, and the effects of surrounding water was assessed with a polarizable continuum model (PCM). To enable a comparison of the overall kinetics for the different reaction mechanisms, thermal rate coefficients and equilibrium constants at selected conditions were calculated from statistical mechanics.

\section{Theoretical methods}

In order to gain information on possible reaction pathways, stationary points on the relevant potential energy surfaces were characterized by systematically optimizing their geometries and validating transition state geometries by intrinsic reaction coordinate calculations [36]. The geometries and harmonic vibrational frequencies of all species were calculated from DFT at the B2PLYP-D3/def2-TZVPP level of theory [37-40] by using the Gaussian 09 program package [41]. The electronic energy contributions were calculated at the $\operatorname{CCSD}\left(\mathrm{F} 12^{\star}\right)\left(\mathrm{T}^{\star}\right) / \mathrm{cc}-\mathrm{pVTZ}$-F12 level of theory [42-44] with the TURBOMOLE 7.3 package of programs [45]. Neither the vibrational frequencies nor the zero-point vibrational energies were scaled because no scaling factors were available for the dispersion-corrected doublehybrid functional used. Also, these scaling factors tend to be very close to unity (for comparison, scaling factor of B2PLYP/def2-TZVPP: $f=0.9977$ [46]).

In order to account for solvation effects from bulk water, we additionally performed DFT calculations at the B2PLYP-D3/def2-TZVPP level of theory [37-40] with the PCM [47, 48], again using the Gaussian 09 program package [41].

The error margins of the obtained reaction energies and energy barriers are assumed to be dominated by the electronic energy contributions and therefore by the accuracy of the employed coupled-cluster method. In a benchmark study by Knizia et al. [39], it was demonstrated that methods like the one used here yield reaction energies with an error margin on the order of $\pm 2 \mathrm{~kJ} \mathrm{~mol}^{-1}$ (mean absolute deviation of $0.833 \mathrm{~kJ} \mathrm{~mol}^{-1}$ for reaction energies calculated at the $\operatorname{CCSD(T)-F12a/AVTZ~level~of~}$ theory [49] multiplied by a conversion factor of 2.5 to obtain the $95 \%$ confidence limit [50]). The PCM calculations were performed at the DFT level and are probably less accurate. A recent review of double-hybrid functionals recommends an error margin of $\pm 2 \mathrm{kcal} \mathrm{mol}^{-1}$ (roughly $\pm 8 \mathrm{~kJ} \mathrm{~mol}^{-1}$ ) for reaction energies from B2PLYP-D3 with a slightly larger quadruple zeta basis set [51]. The semi-empirical PCM contributes an 
additional uncertainty that is difficult to assess. The final results are likely to benefit from error compensation because only relative energies of structurally similar species were calculated. Nonetheless, as the model is essentially classical and semiempirical, an uncertainty on the order of $\pm 20 \mathrm{~kJ} \mathrm{~mol}^{-1}$ seems reasonable. Energies, Cartesian coordinates, rotational constants and harmonic wave numbers of all species are listed in the Supplementary Materials.

It turns out (see below) that reactions 1-3 mostly proceed via (prereactive) reactant complexes and (post reactive) product complexes. For the kinetics and thermodynamics discussion, we use the following lettering for the corresponding sub-steps:

$$
\begin{gathered}
\text { Reactant }(\mathrm{s}) \Leftrightarrow \text { reactant complex } \\
\text { Reactant complex } \rightarrow \text { product complex } \\
\text { Product complex } \rightarrow \text { product }
\end{gathered}
$$

And the following nomenclature for energy barriers: $E_{i b}=E_{i}$ (transition state of step b) $-\mathrm{E}_{i}($ reactant complex $)$ and $E_{i(0)}=\mathrm{E}_{i}\left(\right.$ transition state of step b) $-\mathrm{E}_{i}($ reactant $(\mathrm{s}))$ (reaction $i=1-3, T=0 \mathrm{~K}$ ).

Equilibrium constants for steps a, $K_{i \mathrm{a}}(i=1-3)$, were calculated from statistical thermodynamics (see e. g. [52]) with the program KisThelP [53]. Rate coefficients for steps b, $k_{i \mathrm{~b}}(i=1-3)$, were obtained from canonical transition state theory (see e. $\mathrm{g}$. [54]) in analogy to [55] by using an in-house code. Both programs use the rigid rotor/harmonic oscillator approximation. Tunneling corrections were estimated with the Wigner approximation $[56,57]$ but turned out to be negligible (see below). The resulting kinetic parameterizations are given in the Supplementary Material.

\section{Results and discussion}

\subsection{Formation of biuret}

The formation of biuret in reaction 1 represents the first step of urea condensation considered in the present work. The potential energy diagram of this reaction is shown in Figure 2, and the corresponding transition state structures are schematically depicted in Figure 3. Selected energy values are collected in Table 1. Note that, if not mentioned otherwise, the discussion in the present and subsequent sections will refer to the results from the coupled-cluster calculations.

It turned out that the reaction proceeds via a reactant complex, a result that is in line with the findings in [23]. In the presence of water, also product complexes 


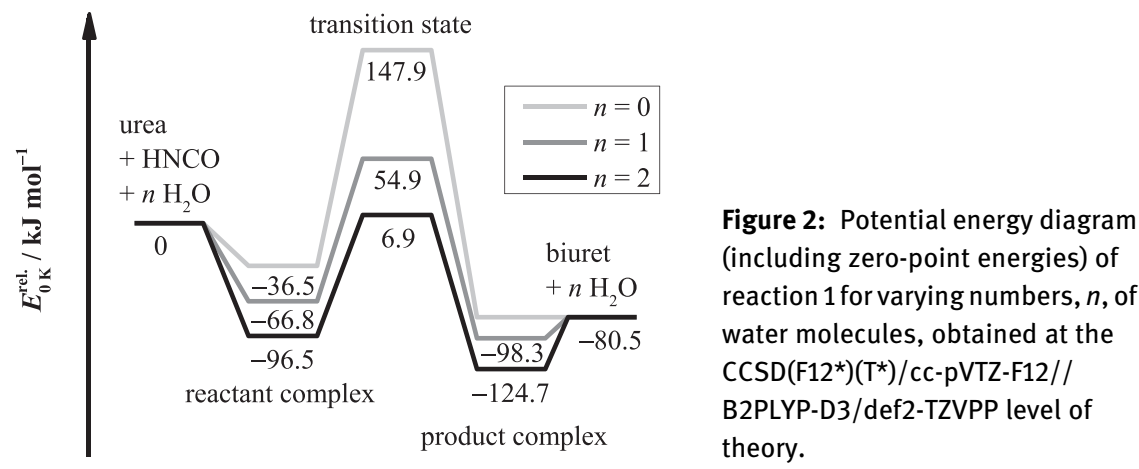

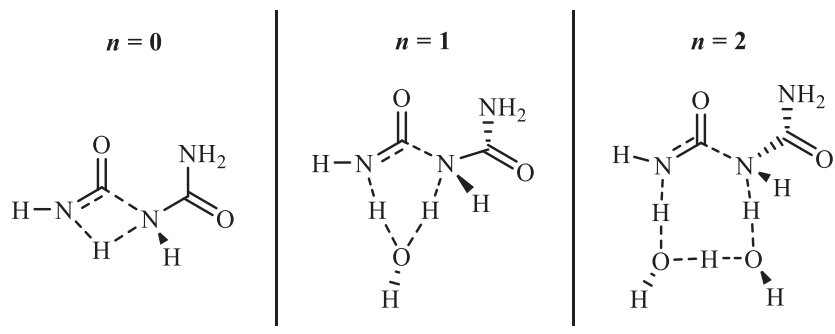

Figure 3: Schematic transition state structures of reaction 1 obtained at the B2PLYP-D3/ def2-TZVPP level of theory with $n$ being the number of water molecules involved.

Table 1: Comparison of reaction energies and threshold energies of reaction 1 obtained in the present work for a different number, $n$, of water molecules, italic numbers including PCM; for quantum chemical methods see text (values for $T=0 \mathrm{~K}$, units: $\mathrm{kJ} \mathrm{mol}^{-1}$ ).

\begin{tabular}{|c|c|c|c|c|c|c|}
\hline & \multicolumn{3}{|c|}{$\operatorname{CCSD}\left(\mathrm{F} 12^{*}\right)\left(\mathrm{T}^{*}\right)$} & \multicolumn{3}{|c|}{ B2PLYP-D3 } \\
\hline & $\Delta E_{1 \mathrm{a}}$ & $E_{1 \mathrm{~b}}$ & $\Delta E_{1 \mathrm{c}}$ & $\Delta E_{1 \mathrm{a}}$ & $E_{1 \mathrm{~b}}$ & $\Delta E_{1 \mathrm{c}}$ \\
\hline \multirow[t]{2}{*}{$n=0$} & -36.5 & 184.4 & - & -39.0 & 193.0 & - \\
\hline & & & & -6.5 & 174.4 & \\
\hline \multirow[t]{2}{*}{$n=1$} & -66.8 & 121.7 & 17.8 & -74.2 & 128.8 & 18.2 \\
\hline & & & & -47.5 & 124.4 & 23.8 \\
\hline \multirow[t]{2}{*}{$n=2$} & -96.5 & 103.4 & 44.2 & -108.6 & 110.1 & 50.4 \\
\hline & & & & -69.3 & 104.2 & 30.3 \\
\hline \multirow[t]{2}{*}{$\Delta_{\mathrm{R}} E_{\mathrm{OK}}$} & -80.5 & & & -71.4 & & \\
\hline & & & & -66.2 & & \\
\hline
\end{tabular}


are found. The overall exoergicity of reaction 1 is $\Delta_{\mathrm{R}} E_{\mathrm{OK}}=\Delta_{\mathrm{R}} H_{\mathrm{OK}}=-80.5 \mathrm{~kJ} \mathrm{~mol}^{-1}$ $\left(-85.2 \mathrm{~kJ} \mathrm{~mol}^{-1}\right.$ in [23])

In the absence of water, the energy barrier of reaction 1 relative to the energy of the reactant complex, $E_{1 \mathrm{~b}}=184.4 \mathrm{~kJ} \mathrm{~mol}^{-1}$, is quite high. The DFT calculations by Sebelius et al. [23] at the B3PW91/6-31++ $\mathrm{G}^{\star \star}$ level of theory yielded an only slightly lower value of $E_{1 \mathrm{~b}}=170 \mathrm{~kJ} \mathrm{~mol}^{-1}$. The DFT calculations of the present work at the B2PLYP-D3/def2-TZVPP level gave $E_{1 \mathrm{~b}}=193.0 \mathrm{~kJ} \mathrm{~mol}^{-1}$. We note here that the transition state geometry is highly strained, due to a four-membered ring structure (see Figure 3, $n=0$ ) and thus energetically demanding. The incorporation of a single water molecule in this reaction system leads to a significant decrease of the energy barrier. The water molecule is mediating the $\mathrm{H}$-atom transfer and leads to a six-membered ring structure in the transition state (see Figure $3, n=1$ ), giving rise to a decrease of the energy barrier by $E_{1 \mathrm{~b}}(n=0)-E_{1 \mathrm{~b}}(n=1)=62.7 \mathrm{~kJ} \mathrm{~mol}^{-1}$ $\left(64.2 \mathrm{~kJ} \mathrm{~mol}^{-1}\right.$ from DFT). If the barriers are counted from the energy level of the reactants, a decrease of $E_{1(0)}(n=0)-E_{1(0)}(n=1)=93.0 \mathrm{~kJ} \mathrm{~mol}^{-1}$ is obtained (99.4 $\mathrm{kJ} \mathrm{mol}^{-1}$ from DFT). Nicolle et al. [34] investigated the decomposition of urea in aqueous solution at the $\operatorname{CCSD}(\mathrm{T})$ level of theory and found a decrease of the energy barrier for the $\mathrm{H}$ transfer by $102 \mathrm{~kJ} \mathrm{~mol}^{-1}$ in going from a four-membered transition state without water to a six-membered transition state with one water molecule. Note that the difference between the ring strain energies of a fourmembered and a (nearly unstrained) six-membered hydrocarbon ring is about $110 \mathrm{~kJ} \mathrm{~mol}^{-1}[58,59]$.

Incorporation of a second water molecule leads to a further but less pronounced lowering of the energy barrier again due to ring strain effects. The remaining energy barrier is $E_{1 \mathrm{~b}}(n=2)=103.4 \mathrm{~kJ} \mathrm{~mol}^{-1}$. In the transition state structure, the $\mathrm{H}$-atom transfer proceeds through an eight-membered ring with nearly right-angular H-O-H-arrangements, involving both water molecules in the process (see Figure 3, $n=2$ ). This further lowering of the energy barrier by $E_{1 \mathrm{~b}}(n=1)$ $-E_{1 \mathrm{~b}}(n=2)=18.3 \mathrm{~kJ} \mathrm{~mol}^{-1}$ is again similar to the effect of two water molecules on the decomposition of urea (lowering of the energy barrier by $11.7 \mathrm{~kJ} \mathrm{~mol}^{-1}$ [34]). Furthermore, the structures of the transition states are similar.

In order to assess the influence of bulk solvation effects, all reaction channels were also characterized with DFT calculations at the B2PLYP-D3/def2-TZVPP level of theory with the PCM of water. The energy values obtained are listed and compared with the gas phase energy values in Table 1 . While in general the absolute values of the stabilization energies of the reactant complexes are smaller, there is no unique trend in the threshold energies and in the stabilization energies of the product complexes.

All in all, the effect of the solvation model on the energy barriers is rather small. Considering the larger error margin of the DFT-level PCM calculations, the 


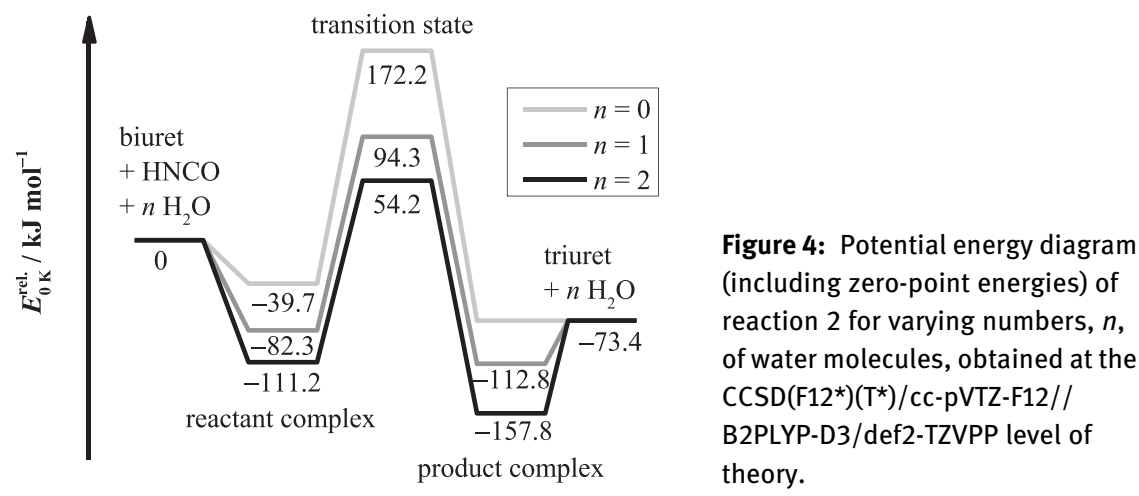

(n)

Figure 5: Schematic transition state structures of reaction 2 obtained at the B2PLYP-D3/ def2-TZVPP level of theory with $n$ being the number of water molecules involved.

Table 2: Comparison of reaction energies and threshold energies of reaction 2 obtained in the present work for a different number, $n$, of water molecules, italic numbers including PCM; for quantum chemical methods see text (values for $T=0 \mathrm{~K}$, units: $\mathrm{kJ} \mathrm{mol}^{-1}$ ).

\begin{tabular}{|c|c|c|c|c|c|c|}
\hline & \multicolumn{3}{|c|}{$\operatorname{CCSD}\left(F 12^{*}\right)\left(T^{\star}\right)$} & \multicolumn{3}{|c|}{ B2PLYP-D3 } \\
\hline & $\Delta E_{2 a}$ & $E_{2 b}$ & $\Delta E_{2 \mathrm{c}}$ & $\Delta E_{2 \mathrm{a}}$ & $E_{2 b}$ & $\Delta E_{2 c}$ \\
\hline \multirow[t]{2}{*}{$n=0$} & -39.7 & 211.9 & - & -41.8 & 215.7 & - \\
\hline & & & & -23.9 & 204.9 & \\
\hline \multirow[t]{2}{*}{$n=1$} & -82.3 & 176.6 & 39.4 & -89.3 & 182.5 & 42.3 \\
\hline & & & & -52.3 & 155.0 & 27.9 \\
\hline \multirow[t]{2}{*}{$n=2$} & -111.2 & 165.4 & 84.4 & -122.3 & 170.9 & 92.5 \\
\hline & & & & -75.2 & 139.0 & 54.9 \\
\hline \multirow[t]{2}{*}{$\Delta_{\mathrm{R}} E_{\mathrm{OK}}$} & -73.4 & & & -63.9 & & \\
\hline & & & & -56.5 & & \\
\hline
\end{tabular}


differences appear not significant. It should also be noted that the continuum model alone is not able to account for any catalytic effect without the additional explicit incorporation of water molecules into the reaction mechanism. Because the threshold energies relative to the reactants, $E_{1(0)}$, are generally higher for the PCM as compared to the gas phase values, even a decrease of the reaction rates is predicted in the presence of liquid water.

\subsection{Formation of triuret}

The addition of isocyanic acid to biuret forms triuret in reaction 2. Figure 4 shows the potential energy diagram and Figure 5 the transition state structures for this reaction. Selected energy values are collected in Table 2.

Again, reactant complexes and, in the presence of water, also product complexes were found. The overall exoergicity of reaction 2 is $\Delta_{\mathrm{R}} E_{\mathrm{OK}}=\Delta_{\mathrm{R}} H_{\mathrm{OK}}=$ $-73.4 \mathrm{~kJ} \mathrm{~mol}^{-1}\left(-60.9 \mathrm{~kJ} \mathrm{~mol}^{-1}\right.$ in [23]). The reaction channel without water exhibits the highest energy barrier $E_{2 \mathrm{~b}}=211.9 \mathrm{~kJ} \mathrm{~mol}^{-1}$. The analogous value from Sebelius et al. [23] is $177 \mathrm{~kJ} \mathrm{~mol}^{-1}$. The stabilization energy of the reactant complex obtained in the present work is $\Delta E_{2 \mathrm{a}}=-39.7 \mathrm{~kJ} \mathrm{~mol}^{-1}$ compared to a value of ca. $-20 \mathrm{~kJ} \mathrm{~mol}^{-1}$ inferred from Figure 9 of [23].

Incorporation of one and two mediating water molecules lowers the energy barrier by $E_{2 \mathrm{~b}}(n=0)-E_{2 \mathrm{~b}}(n=1)=35.3 \mathrm{~kJ} \mathrm{~mol}^{-1}$ and $E_{2 \mathrm{~b}}(n=0)-E_{2 \mathrm{~b}}(n=2)=46.5 \mathrm{~kJ} \mathrm{~mol}^{-1}$, respectively. Obviously, in analogy to reaction 1 , the strain of the ring structure in the transition state is reduced by the increased ring size. But this catalytic effect is less pronounced than in reaction 1 .

The results of the PCM calculations, listed in Table 2, exhibit a similar trend, but compared to reaction 1 , the differences are larger. While the energy barriers without explicit involvement of a water molecule are quite similar, the energy barriers obtained from PCM are considerably lower for one or two mediating water molecules. This is due to the fact that the transition states of reaction 2 have larger dipole moments and hence are more stabilized by continuum solvation effects.

\subsection{Formation of cyanuric acid}

The cyclization of triuret yields cyanuric acid upon elimination of ammonia, reaction 3. The corresponding potential energy diagram is shown in Figure 6, and the transition state structures are sketched in Figure 7. Selected energy values are collected in Table 3. 


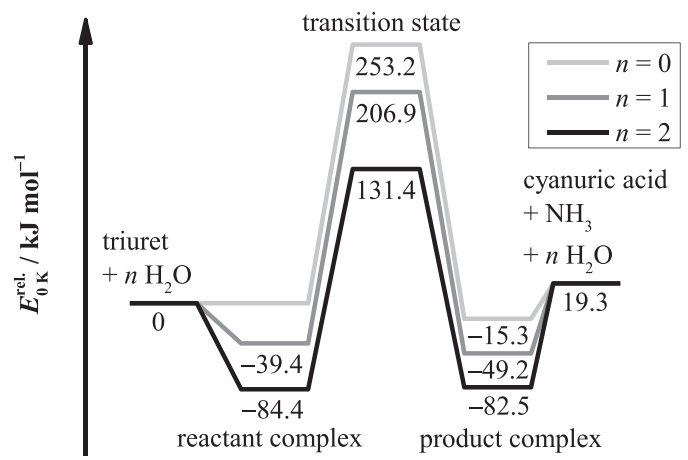

Figure 6: Potential energy diagram (including zero-point energies) of reaction 3 for varying numbers, $n$, of water molecules, obtained at the CCSD $\left(F 12^{\star}\right)\left(\mathrm{T}^{\star}\right) /$ cc-pVTZ-F12// B2PLYP-D3/def2-TZVPP level of theory.

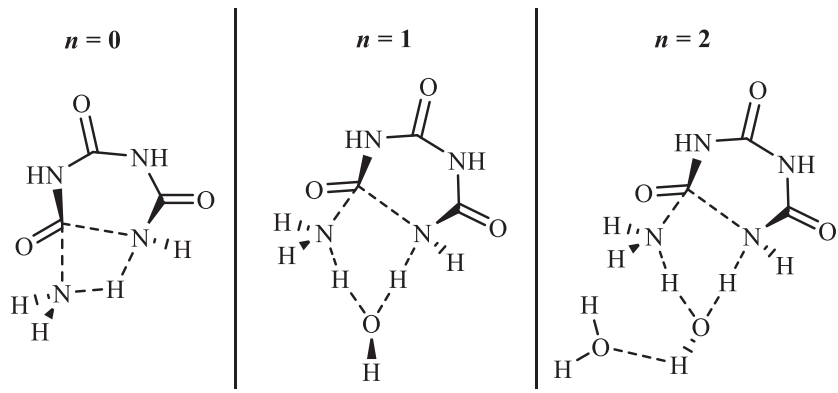

Figure 7: Schematic transition state structures of reaction 3 obtained at the B2PLYP-D3/ def2-TZVPP level of theory with $n$ being the number of water molecules involved.

Table 3: Comparison of reaction energies and threshold energies of reaction 3 obtained in the present work for a different number, $n$, of water molecules, italic numbers including PCM; for quantum chemical methods see text (values for $T=0 \mathrm{~K}$, units: $\mathrm{kJ} \mathrm{mol}^{-1}$ ).

\begin{tabular}{|c|c|c|c|c|c|c|}
\hline & \multicolumn{3}{|c|}{$\operatorname{CCSD}\left(\mathrm{F} 12^{\star}\right)\left(\mathrm{T}^{\star}\right)$} & \multicolumn{3}{|c|}{ B2PLYP-D3 } \\
\hline & $\Delta E_{3 a}$ & $E_{3 b}$ & $\Delta E_{3 c}$ & $\Delta E_{3 a}$ & $E_{3 b}$ & $\Delta E_{3 c}$ \\
\hline \multirow[t]{2}{*}{$n=0$} & - & 253.2 & 34.6 & - & 251.0 & 31.1 \\
\hline & & & & & 218.5 & 7.4 \\
\hline \multirow[t]{2}{*}{$n=1$} & -39.4 & 246.3 & 68.5 & -42.3 & 247.1 & 74.6 \\
\hline & & & & -27.9 & 223.4 & 52.7 \\
\hline \multirow[t]{2}{*}{$n=2$} & -84.4 & 215.8 & 101.8 & -92.5 & 223.6 & 111.4 \\
\hline & & & & -54.9 & 212.5 & 80.3 \\
\hline \multirow[t]{2}{*}{$\Delta_{\mathrm{R}} E_{0 \mathrm{~K}}$} & 19.3 & & & 20.4 & & \\
\hline & & & & 19.3 & & \\
\hline
\end{tabular}




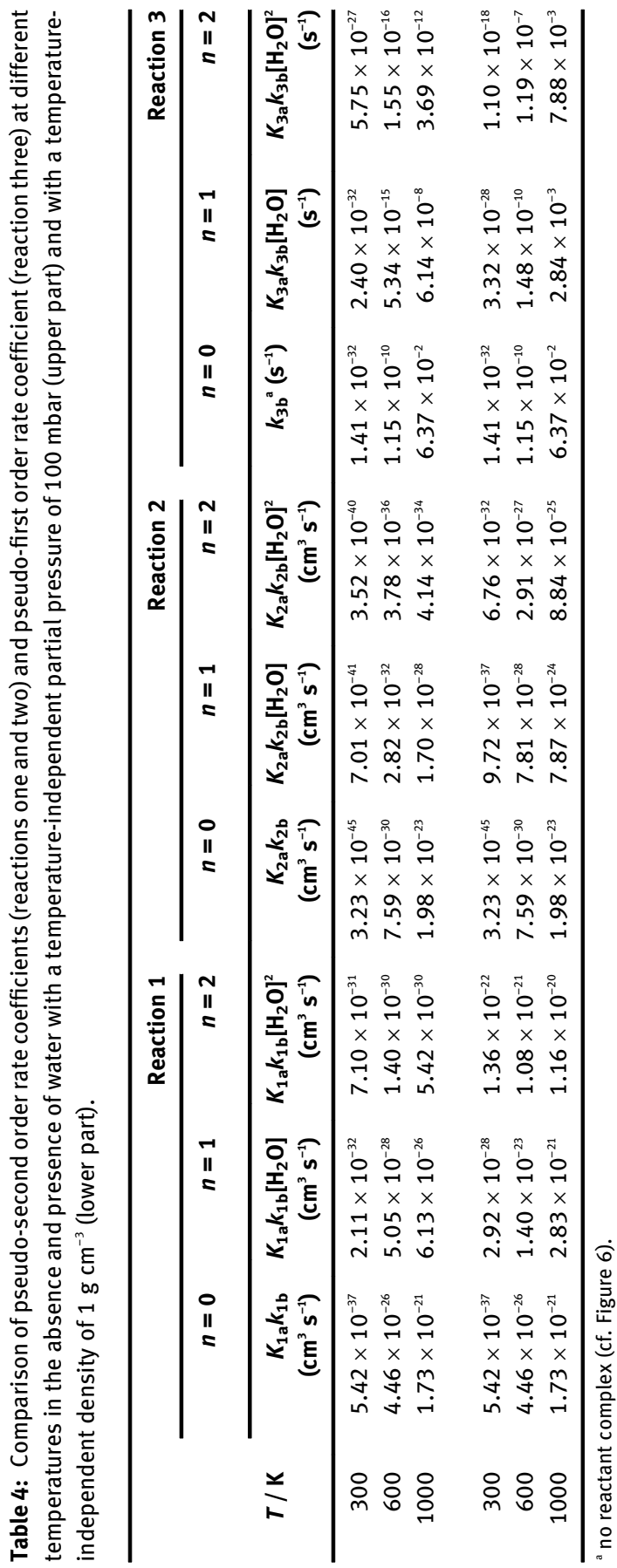


Except in the absence of water, reactant complexes are formed, whereas product complexes occur with and without water. The overall endoergicity of reaction 3 is $\Delta_{\mathrm{R}} E_{\mathrm{OK}}=\Delta_{\mathrm{R}} H_{\mathrm{OK}}=19.3 \mathrm{~kJ} \mathrm{~mol}^{-1}$ Here, a direct comparison with the values from [23] is not possible because the structures shown in Figure 9 of [23] are only in part consistent with those of the present work, and more detailed information is unfortunately not given. The reaction energy relative to the all-trans conformation of triuret inferred from Figure 9 of [23] is on the order of $130 \mathrm{~kJ} \mathrm{~mol}^{-1}$ that is ca. $110 \mathrm{~kJ} \mathrm{~mol}^{-1}$ above the value of the present work.

In the absence of water, the overall energy barrier is quite high with a value of $253.2 \mathrm{~kJ} \mathrm{~mol}^{-1}$ (note that there is no reactant complex in this case). The corresponding value from Sebelius et al. [23] is ca. $240 \mathrm{~kJ} \mathrm{~mol}^{-1}$ and thus in reasonable agreement.

Inclusion of one water molecule decreases the energy barrier counted from the reactants, $E_{3(0)}$, by $46.3 \mathrm{~kJ} \mathrm{~mol}^{-1}$, and inclusion of a second water molecule leads to a further decrease by $75.5 \mathrm{~kJ} \mathrm{~mol}^{-1}$. Nonetheless, the remaining energy barriers still remain quite high, ruling out this mechanism as a pathway to cyanuric acid under SCR conditions in the exhaust tract (see below).

Table 3 also contains the energy values obtained with the PCM. A considerable decrease of the threshold energy $E_{3 \mathrm{~b}}$ by $32.5 \mathrm{~kJ} \mathrm{~mol}^{-1}$ in going from the gas phase to the solvent phase for $n=0$ occurs (note that there is no reactant complex). Within the PCM, addition of one or two water molecules has only a small effect on $E_{3 \mathrm{~b}}$ without a distinct trend. Also the enthalpy of reaction is hardly changed. Obviously, the solvation effects as described by the PCM tend to cancel for reactants and products.

\subsection{Comparison of the electronic structure calculations}

From Tables 1-3 it becomes evident that the relative energies of the stationary points along the reaction coordinates obtained with the coupled-cluster $\left(\mathrm{CCSD}\left(\mathrm{F} 12^{\star}\right)\left(\mathrm{T}^{\star}\right) / \mathrm{cc}-\mathrm{pVTZ}-\mathrm{F} 12 / / \mathrm{B} 2 \mathrm{PLYP}-\mathrm{D} 3 /\right.$ def2-TZVPP) and the density functional (B2PLYP-D3/def2-TZVPP//B2PLYP-D3/def2-TZVPP) theory calculations for the gas phase are in reasonable agreement. With the exceptions of $\Delta E_{1 \mathrm{a}}$ and $\Delta E_{2 \mathrm{a}}$ for $n=2$, the differences are below $10 \mathrm{~kJ} \mathrm{~mol}^{-1}$, often much less. The qualitative trends for an increasing number of water molecules are correctly predicted by both methods for all three reactions.

Adding the corrections from the PCM does not change the energetic order of the stationary points except for reaction $1 \mathrm{~b}(n=1,2)$ and reaction $3 \mathrm{~b}(n=0,1)$. 


\subsection{Structure analogy considerations}

A comparison of the structures in Figures 3, 5 and 7 shows that all three reactions proceed via transition states with similar ring structures for $n=0$ and $n=1$. These transition states contain nearly planar four- and six-membered rings, respectively, with similar structural parameters that are indicated by the dashed lines. Due to the cyclic structure of the reaction product, the ring structure of the transition state in reaction 3 for $n=1$ is somewhat stronger distorted as those of reactions 1 and two. Also the types of the bonds that are broken or formed, on the one hand, in reactions 1 and 2 and, on the other hand, in reaction 3 are in part different.

A comparison of the transition state structures for $n=2$ reveals a different trend. While the structural features remain similar for the transition states of reactions one and two, in which the $\mathrm{H}$-atom transfers proceed via eight-membered rings, the transition state of reaction 3 contains only a six-membered ring. Here, only one water molecule mediates the $\mathrm{H}$-atom transfer whereas the second water molecule acts as a spectator and merely stabilizes the transition state through a hydrogen bond. No indications for a transition state in which the H-atom transfer involves both water molecules could be found. Conversely, no low-lying transition states with spectator water molecules were found for reactions 1 and 2.

These structural differences in the transition states provide a possible explanation for the less pronounced lowering of the energy barrier $E_{i(0)}$ for a growing number of water molecules in reaction 3 compared to reactions 1 and two. The energetic effect of water molecules on the $\mathrm{H}$-atom transfer is probably smaller for a cyclization reaction like reaction three.

\subsection{Kinetic considerations}

In order to evaluate the effect of water molecules on the kinetics of reaction 1-3, pseudo-second order (reactions 1 and two) and pseudo-first order (reaction three) rate coefficients were calculated. In view of the substantial barrier heights, the rate coefficients were assumed to be in the high-pressure limit under most practical conditions and the pre-equilibria between reactants and reactant complexes to be established. At nearly all temperatures, tunneling correction factors between 1 and 2 were obtained from the Wigner approximation. Given the facts that this approach often overestimates tunneling effects [55], and that the rate coefficients to be compared differ by many orders of magnitude (cf. Table 4), we decided not to apply more elaborated tunneling models but to neglect these small corrections.

With these assumptions, pseudo-second order rate coefficients of the form $K_{i \mathrm{a}} k_{i b}\left[\mathrm{H}_{2} \mathrm{O}\right]^{n}$ for reaction $i=1$ or 2 , with $n=0$, 1 , or 2 being the number of water 
molecules involved, follow from elementary kinetic considerations. Analogously, for reaction 3 pseudo-first order rate coefficients, $K_{3 \mathrm{a}} \mathrm{k}_{3 \mathrm{~b}}\left[\mathrm{H}_{2} \mathrm{O}\right]^{n}$, are obtained with formally $K_{3 \mathrm{a}}=1$ in the case of $n=0$ (no reactant complex, cf. Figure 6). The equilibrium constants and rate coefficients were calculated as outlined in section 2, and illustrative results for given water concentrations are collected in Table 4. In the upper part of Table 4, a water concentration corresponding to a partial pressure of $p\left(\mathrm{H}_{2} \mathrm{O}\right)=100$ mbar at the actual temperature was used whereas for the lower part a liquid-like density of $1 \mathrm{~g} \mathrm{~cm}^{-3}$ independent of temperature was assumed. Note that these water concentrations (though they might reflect certain conditions in the urea-SCR process) were chosen artificially as a reference to be used for the calculation of pseudo-first/second order rate coefficients to enable the comparison of reaction rates for different mechanisms. Results for other temperatures as well as parameterizations of the temperature dependence of the rate coefficients and equilibrium constants are listed in the Supplementary Material.

From Table 4 it becomes obvious that all the pseudo-first and pseudo-second order rate coefficient increase with increasing temperature, often by many orders of magnitude. This is reasonable and directly related to the apparent energies of activation, which are essentially governed by the threshold energies relative to the corresponding reactants, $E_{i(0)}$. These threshold energies $E_{i(0)}$ are larger than zero for all the considered reactions and vary between $6.9 \mathrm{~kJ} \mathrm{~mol}^{-1}(i=1, n=2$, cf. Figure 2) and $253.2 \mathrm{~kJ} \mathrm{~mol}^{-1}(i=3, n=0$, cf. Figure 6). The temperature dependence of the overall rate coefficients is correspondingly weak or strong.

The dependence on the number of water molecules participating in the reactions is less clear-cut. Complications arise because the pseudo-first and pseudo-second order rate coefficients to be compared contain the actual water concentration. We first note that the threshold energies $E_{i b}$ generally decrease with an increasing number of water molecules. Consequently, the unimolecular isomerization step, reaction $b$, is generally accelerated. But this effect may be overcompensated by a lower concentration of the reactant complex for not sufficiently high water concentrations. The extent of this lowering depends on the change of the well depth due to the additional water molecules but also on the specific water concentrations chosen. So, a generally valid tendency cannot be given here.

Inspection of Table 4 (see also Tables S20 and S21 in the Supplemental Material) reveals that for $p\left(\mathrm{H}_{2} \mathrm{O}\right)=100$ mbar, that is $\left[\mathrm{H}_{2} \mathrm{O}\right] \approx 10^{18} \mathrm{~cm}^{-3}$, the rate coefficients increase for an increasing number of water molecules only at the lowest temperatures near $300 \mathrm{~K}$. At the higher temperatures, the rate coefficients generally decrease. The situation changes for the higher water concentration, $\left[\mathrm{H}_{2} \mathrm{O}\right] \approx 3 \times 10^{22} \mathrm{~cm}^{-3}$ corresponding to $\rho=1 \mathrm{~g} \mathrm{~cm}^{-3}$, where the rate coefficients increase at nearly all temperatures except at the highest temperatures in the case of reactions 2 and 3. 
In the following, some conclusions regarding the urea-SCR process will be drawn. On the basis of typical mass flow rates and dimensions of a diesel engine exhaust tract [60], we assume maximum reaction times on the order of $100 \mathrm{~ms}$. Under the conditions of Table 4, the lowest half-life from the (pseudo-)first order rate coefficients of reaction $3, t_{1 / 2}=\ln 2 / k_{3 \mathrm{~b}}=11 \mathrm{~s}$, arises for $n=0$ and $T=1000 \mathrm{~K}$. Because even this minimum value is two orders of magnitude larger than the maximum reaction time assumed above, reaction 3 can be considered unimportant under diesel exhaust conditions. To estimate half-lives for reactions 1 and 2, we use the simplifying assumption of a (pseudo-)second order reaction $\mathrm{A}+\mathrm{B}$ with equal initial concentrations, $[A]_{0}=[B]_{0}$. It turns out that with the overall rate coefficients from Table 4 it would take unreasonably high initial reactant concentrations to obtain half-lives as low as $100 \mathrm{~ms}$. Even for the most favorable conditions (Reaction $1,\left[\mathrm{H}_{2} \mathrm{O}\right] \approx 3 \times 10^{22} \mathrm{~cm}^{-3}$, $T=1000 \mathrm{~K}, n=2)$, this requires $[\mathrm{urea}]_{0}=[\mathrm{HNCO}]_{0}=\left(t_{1 / 2} K_{1 \mathrm{a}} k_{1 \mathrm{~b}}\left[\mathrm{H}_{2} \mathrm{O}\right]^{2}\right)^{-1}=8.6 \times 10^{20} \mathrm{~cm}^{-3}$, which formally corresponds to extremely high partial pressures of $p_{0}$ (urea $)=p_{0}(\mathrm{HNCO})=120$ bar. By considering that the total pressure in the exhaust tract typically varies between 1 and 5 bar [61], it becomes obvious that also reactions 1 and 2 do not play any role under these conditions.

\section{Summary and conclusion}

In the present work, the kinetics of three different reactions of urea and its condensation products were investigated by employing high-level quantum chemical methods and statistical rate theory. It could be demonstrated that water has a catalytic effect on these reactions. Water molecules influence the microscopic mechanisms by extending the ring size of the transition states and mediating the hydrogen atom transfer. Water molecules also participate in the formation of the pre-reactive complexes. Accordingly, the overall kinetics is complicated and strongly dependent on the absolute water concentration. The mere presence of bulk water simulated with a PCM had no additional effect. Under conditions typical for the urea-SCR process, these homogeneous reactions are too slow, however, to explain the observed formation of urea condensation products. Other, probably ionic or radical reactions have to be considered.

Acknowledgment: Funded by the Deutsche Forschungsgemeinschaft (DFG, German Research Foundation) - Projektnummer 237267381 - TRR 150. The authors also acknowledge generous allocation of computer time by the state of Baden-Württemberg through bwHPC and the DFG through grant no INST 40/467-1 FUGG (JUSTUS cluster). 
Author contribution: All the authors have accepted responsibility for the entire content of this submitted manuscript and approved submission.

Research funding: This research was funded by the Deutsche Forschungsgemein schaft (DFG, German Research Foundation) - Projektnummer 237267381 - TRR 150.

Conflict of interest statement: The authors declare no conflicts of interest regarding this article.

\section{References}

1. Noval., TronconiE., Eds. Urea-SCR Technology for deNOx After Treatment of Diesel Exhausts; Springer: New York, 2014.

2. Granger P., Parvulescu V. I. Chem. Rev. 2011, 111, 3155.

3. Lundström A., Snelling T., Morsing P., Gabrielsson P., Senar E., Olsson L. Appl. Catal. B. 2011, 106, 273.

4. Bernhard A. M., Peitz D., Elsener M., Wokaun A., Kroecher O. Appl. Catal. B. 2012, 115-116, 129.

5. Bernhard A. M., Peitz D., Elsener M., Schildhauer T., Kröcher O. Catal. Sci. Technol. 2013, 3, 942.

6. Ebrahimian V., Nicolle A., Habchi C. AlChE J. 2011, 58, 1998.

7. Brack W., Heine B., Birkhold F., Kruse M., Schoch G., Tischer S., Deutschmann O. Chem. Eng. Sci. 2014, 106, 1.

8. Tischer S., Börnhorst M., Amsler J., Schoch G., Deutschmann O. Phys. Chem. Chem. Phys. 2019, 21, 16785.

9. Börnhorst M., Langheck S., Weickenmeier H., Dem C., Suntz R., Deutschmann O. Chem. Eng. J. 2019, 377, 119855.

10. Koebel M., Elsener M., Marti T. Combust. Sci. Technol. 1996, 121, 85.

11. Seker E., Yasyerli N., Gulari E., Lambert C., Hammerle R. H. Appl. Catal. B. 2002, 37, 27.

12. Stradella L., Argentero M. Thermochim. Acta. 1993, 219, 315.

13. Schaber P. M., Colson J., Higgins S., Thielen D., Anspach B., Brauer J. Thermochim. Acta. 2004, 424, 131.

14. Eichelbaum M., Farrauto R. J., Castaldi M. J. Appl. Catal. B. 2010, 97, 90.

15. Fawsitt C. E. Z. Phys. Chem. 1902, 41, 601.

16. Werner E. A. J. Chem. Soc. Trans. 1918, 113, 84.

17. Warner R. C. J. Biol. Chem. 1942, 142, 705.

18. Shaw W. H. R., Bordeaux J. J. J. Am. Chem. Soc. 1955, 77, 4729.

19. Wolf B., Horsch W. Pharmazie. 1986, 41, 485.

20. Gratzfeld D., Olzmann M. Chem. Phys. Lett. 2017, 679, 219.

21. Dorofeeva O. V., Suchkova T. A. J. Chem. Thermodyn. 2019, 131, 254.

22. Jeilani Y. A., Orlando T. M., Pope A., Pirim C., Nguyen M. T. RSC Adv. 2014, 4, 32375.

23. Sebelius S., Le T. T., Pettersson L. J., Lind H. Chem. Eng. J. 2013, 231, 220.

24. Peitz D., Bernhard A., Kröcher O., In: Urea-SCR Technology for deNOx After Treatment of Diesel Exhausts; Nova I., Tronconi E., Eds. Springer: New York, 2014; pp. 485.

25. Buszek R. J., Francisco J. S., Anglada J. M. Int. Rev. Phys. Chem. 2011, $30,335$.

26. Hofmann M., Schleyer P. v. R. J. Am. Chem. Soc. 1994, 116, 4947.

27. Morokuma K., Muguruma C. J. Am. Chem. Soc. 1994, 116, 10316. 
28. Kallies B., Mitzner R. J. Mol. Model. 1998, 4, 183.

29. Tsipis C. A., Karipidis P. A. J. Am. Chem. Soc. 2003, 125, 2307.

30. Estiu G., Merz K. M. J. Am. Chem. Soc. 2004, 126, 6932.

31. Alexandrova A. N., Jorgensen W. L. J. Phys. Chem. B. 2007, 111, 720.

32. Yao M., Chen X., Zhan C.-G. Chem. Phys. Lett. 2015, 625, 143.

33. Yao M., Tu W., Chen X., Zhan C.-G. Org. Biomol. Chem. 2013, 11, 7595.

34. Nicolle A., Cagnina S., de Bruin T. Chem. Phys. Lett. 2016, 664, 149.

35. Borduas N., Place B., Wentworth G. R., Abbatt J. P. D., Murphy J. G. Atmos. Chem. Phys. 2016, $16,703$.

36. Fukui K. Acc. Chem. Res. 1981, 14, 363.

37. Grimme S. J. Chem. Phys. 2006, 124, 034108.

38. Grimme S., Antony J., Ehrlich S., Krieg H. J. Chem. Phys. 2010, 132, 154104.

39. Weigend F., Ahlrichs R. Phys. Chem. Chem. Phys. 2005, 7, 3305.

40. Weigend F. Phys. Chem. Chem. Phys. 2006, 8, 1057.

41. Frisch G. W. T. M. J., Schlegel H. B., Scuseria G. E., Robb M. A., Cheeseman J. R., Scalmani G., Barone V., Mennucci B., Petersson G. A., Nakatsuji H., et al. Gaussian 09, Revision D.01; Gaussian, Inc.: Wallingford CT, 2013.

42. Hättig C., Tew D. P., KöhnA. J. Chem. Phys. 2010, 132, 231102.

43. Bachorz R. A., Bischoff F. A., Glöß A., Hättig C., Höfener S., Klopper W., Tew D. P. J. Comput. Chem. 2011, 32, 2492.

44. Peterson K. A., Adler T. B., Werner H.-J. J. Chem. Phys. 2008, 128, 084102.

45. TURBOMOLE V73 2018, A development of University of Karlsruhe and Forschungszentrum Karlsruhe GmbH, 1989-2007, TURBOMOLE GmbH, since 2007; available from http://www. turbomole.com.

46. Kesharwani M. K., Brauer B., Martin J. M. L. J. Phys. Chem. A. 2015, 119, 1701.

47. Miertuš S., Scrocco E., Tomasi J. Chem. Phys. 1981, 55, 117.

48. Tomasi J., Mennucci B., Cammi R. Chem. Rev. 2005, 105, 2999.

49. Knizia G., Adler T. B., Werner H.-J. J. Chem. Phys. 2009, 130, 054104.

50. Ruscic B. Int. J. Quantum Chem. 2014, 114, 1097.

51. Goerigk L., Grimme S. Wires Comput. Mol. Sci. 2014, 4, 576.

52. Hill T. L. An Introduction to Statistical Thermodynamics; Addison-Wesley: Reading, MA, 1960.

53. Canneaux S., Bohr F., Henon E. J. Comput. Chem. 2014, 35, 82.

54. Glasstone S., Laidler K. J., Eyring H. The Theory of Rate Processes; McGraw-Hill: New York, 1941.

55. Bänsch C., Kiecherer J., Szöri M., Olzmann M. J. Phys. Chem. A. 2013, 117, 8343.

56. Wigner E. Z. Phys. Chem. 1932, B19, 203.

57. Henriksen N. E., Hansen F. Y. Theories of Molecular Reaction Dynamics; Oxford University Press: Oxford 2008.

58. Benson S. Thermochemical Kinetics, 2nd ed.; Wiley: New York, 1976.

59. Viskolcz B., Lendvay G., Seres L. J. Phys. Chem. A. 1997, 101, 7119.

60. Heywood J. B. Internal Combustion Engine Fundamentals; McGraw-Hill: New York, 1988.

61. Castillo F., Witrant E., Dugard L., Talon V. SAE Tech. Pap. 2013 2013-01-1752.

Supplementary material: The online version of this article offers supplementary material https://doi.org/10.1515/zpch-0000-0000 